\title{
Hale cycle in solar-rotation related recurrence of galactic cosmic rays
}

\section{Agnieszka Gil*}

Institute of Mathematics and Physics, Siedlce University, 3 Maja 54, 08-110 Siedlce, Poland

E-mail: gila@uph.edu.pl

\section{Kalevi Mursula}

Space Climate Research Unit, University of Oulu, FIN-90014 Finland

E-mail: kalevi.mursula@oulu.fi

\begin{abstract}
We study the long-term change of the amplitude of the first (27-day, $A_{27}$ ) and second (14-day, $\left.A_{14}\right)$ harmonics of the galactic cosmic rays (GCR) recurrent variation connected to the solar rotation period. Using two high-latitude neutron monitors, Oulu and Apatity, we verify the dominant 11-year cycle in $A_{27}$ and $A_{14}$ and their systematic 22-year variation (Hale cycle) during sunspot minima, with higher values during the positive polarity $(\mathrm{A}>0)$ minima than during negative (A $<0)$. We show that the $A_{27}$ and $A_{14}$ amplitudes during sunspot minima exhibit a slight declining trend. We suggest that this declining trend is due to the weakening of the solar polar magnetic field during the previous four solar cycles and the ensuing latitudinal widening of the heliospheric current sheet (HCS) region. After trend removal the amplitudes of the 22-year variation increase, and are typically about $15-30 \%$ for $A_{27}$ and about 30-45\% for $A_{14}$. Here we quantify the 22-year cyclicity in $A_{27}$ and $A_{14}$ in a robust way for last five solar minima and give a physical explanation to the earlier ideas about the effect of drifts to produce the observed phase of the 22-year cyclicity.
\end{abstract}




\section{Introduction}

The periodicity of 27 days due to the synodic solar rotation is detected in several parameters of solar activity and in solar wind (SW). The recurrence of galactic cosmic ray (GCR) flux with the rotation of the Sun was first indicated by Forbush [1] by the aid of ionization chambers. Studying data during 1954-1998, from neutron monitors and spacecraft Richardson and coauthors [2] showed the existence of a 22-year Hale cycle in the amplitude of the 27-day recurrence of GCR. The Hale cycle consists of two successive 11-year Schwabe cycles of opposite magnetic field polarities [3]. Alania and coauthors $[4,5]$ verified that the amplitudes of the first and the second harmonics of GCR recurrence are greater during positive polarity minima $(A>0$, magnetic field lines directed outward from the North pole) than negative $(A<0$, magnetic field lines directed outward from the South).

Kota and Jokipii [6], by a non-stationary three dimensional model of GCR transport, containing a southward shifted heliospheric current sheet and corotating interaction regions (CIRs), confirmed the polarity dependence of rotation related quasi-periodic variations of GCR intensity. Iskra and coauthors [7] proposed that the 27-day recurrence of GCR flux has a greater amplitude during $A>0$ minimum epochs because the drift stream has the identical direction as convection stream during those times, but are oppositely directed during $A<0$. They recognized the 27-day recurrence of GCR flux due to the heliolongitudinal asymmetry of solar wind in the inner heliosphere.

Dunzlaff and coauthors [8] suggested that coronal holes structures differ in $A>0$ and $A<0$ minimum epochs, bringing to a 22-year variation in CIRs. Alania and coauthors [9] studied the distribution of the 27-day variation phase of solar wind speed and found more stable and long-lived heliolongitudinal configurations during $A>0$ minimum epochs, probably affecting the amplitude of 27-day variation of cosmic rays flux and producing the observed Hale cycle dependence.

In this paper we calculate the amplitudes of GCR flux variation during the full synodic solar rotation period $\left(A_{27}\right)$ and half rotation period $\left(A_{14}\right)$ in 1964-2017. We find that both amplitudes show a strong 22-year Hale cycle on top of leading 11-year solar cycle, with both amplitudes being greater during positive than negative minimum epochs. We show that both $A_{27}$ and $A_{14}$ exhibit a declining trend during solar minima, which is most probably linked to the weakening of the solar polar magnetic fields in the previous four solar cycles.

\section{Data and methods}

Since the amplitude of the rotation related GCR intensity variation is more pronounced in the lower cutoff-rigidity stations [10,11], and neutron monitors measure the lower energy cosmic rays between $1-50 \mathrm{GeV}[12,13]$, we analyze cosmic ray measured by two high-latitudinal stations, Apatity (lat. $67.57^{\circ} \mathrm{N}$, effective vertical cutoff-rigidity of $0.65 \mathrm{GV}$; data retrieved from pgia.ru and nmdb.eu) and Oulu $\left(65.05^{\circ} \mathrm{N}, 0.8 \mathrm{GV}\right.$; cosmicrays.oulu.fi). We consider the time interval from 1964 to 2017 (in 2017 only half a year), and study not only the 22-year variability but also the long-term trend in rotation related variation of cosmic ray intensity. Using daily data from the two above detectors, we computed the two harmonic amplitudes $A_{27}$ and $A_{14}$ as follows [14]. For each consecutive 27-day period we calculated the amplitude for the first $A_{27}(\mathrm{k}=1)$ and second $A_{14}$ $(\mathrm{k}=2)$ harmonics. After calculating the amplitudes for each rotation we have excluded from further 
consideration all solar rotations affected by Forbush decreases [15, 16, 17]. After that, in order to emphasize the long-term evolution and to eliminate short-term disturbances, we have calculated the yearly means of the two amplitudes and smoothed them with a three-year running mean.
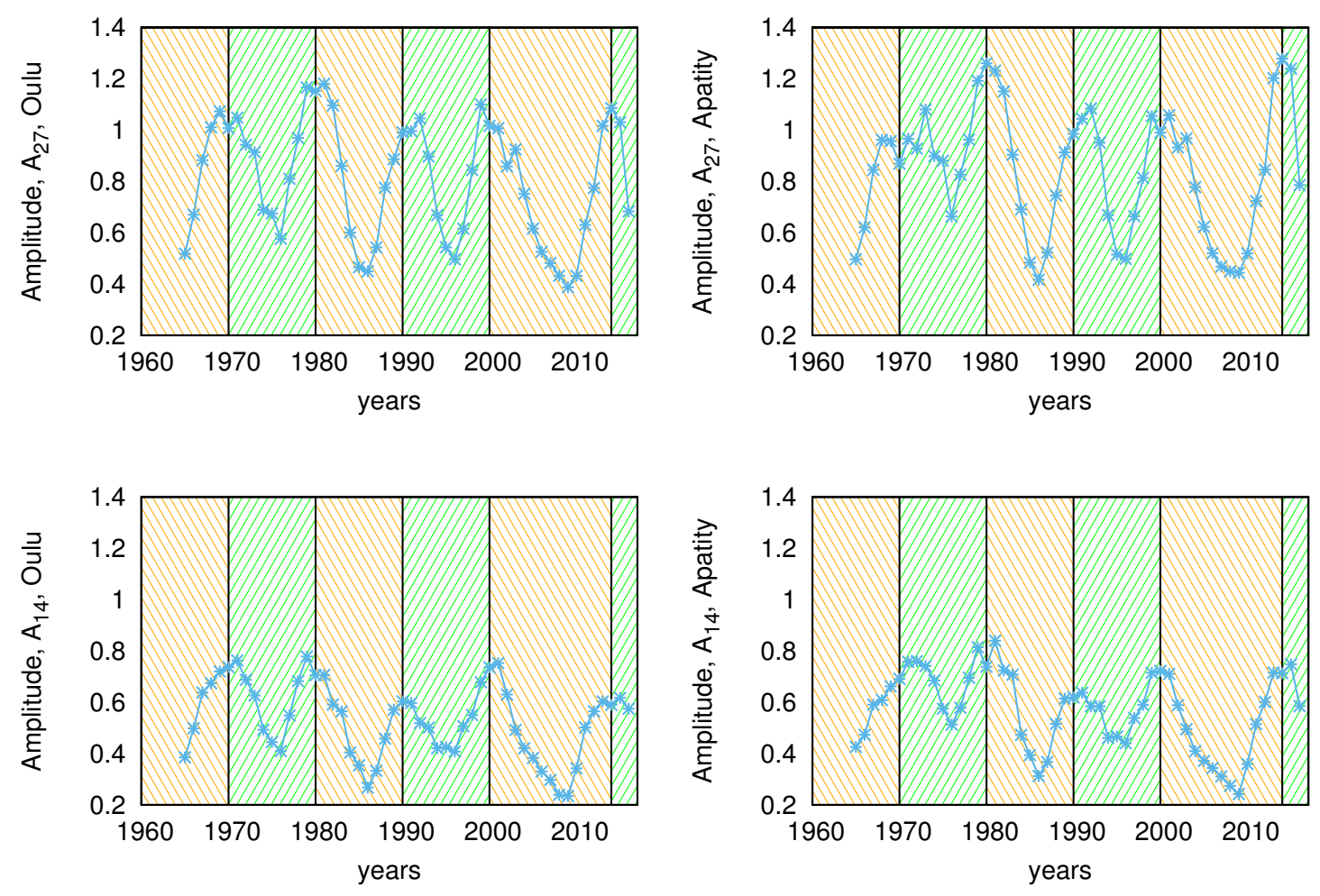

Figure 1: Three-year running means of yearly averaged amplitudes of the first $\left(A_{27}\right.$, upper panels $)$ and the second ( $A_{14}$, lower panels) harmonics of GCR for Oulu (left panels) and Apatity (right panels) neutron monitor count rates in 1964-2017 during $A>0$ epochs (green boxes with upward lattice) and $A<0$ epochs (orange boxes with downward lattice).

\section{Results and discussion}

Figure 1 depicts the amplitudes of the first $\left(A_{27}\right)$ and the second $\left(A_{14}\right)$ harmonics of GCR intensity variation for Oulu and Apatity neutron monitors. Figure 1 illustrates a dominant 11year cycle in $A_{27}$ and $A_{14}$ with minima around solar minima and maxima around solar maxima [18]. Still, on top of this 11-year cycle there is a regular 22-year variation in the level of both amplitudes around solar minimum epochs, confirming the above discussed polarity dependence $[2,5]$. The both harmonics amplitudes of GCR intensity variation are greater during each $A>0$ polarity minimum than the corresponding amplitudes during the previous or the following $A<0$ polarity minima. During maximum times of solar activity the 22-year cycle in $A_{27}$ or $A_{14}$ amplitude is not as organized as during solar minima because other, more important factors than drifts disturb the variation of GCR at these times. 

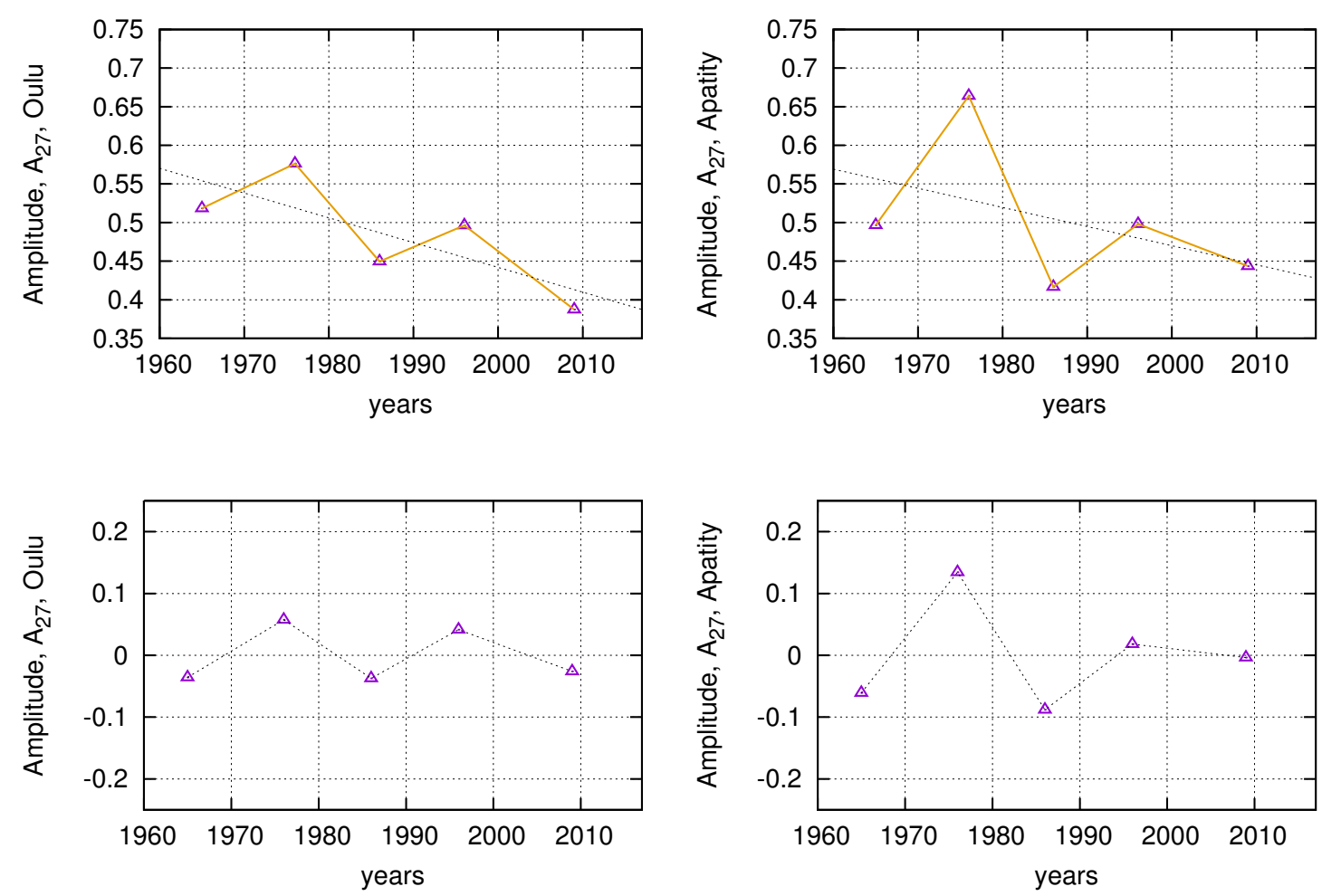

Figure 2: Three-year means of amplitude of the first $\left(A_{27}\right)$ harmonic of GCR recurrence for the last five solar minima with linear trend (upper panels) and detrended (lower panels) for Oulu (left panels) and Apatity (right panels) neutron monitor count rates.

The upper panels of Figures 2 and 3 present the lowest values of $A_{27}$ and $A_{14}$, respectively, for the two NMs, for all solar minimum epochs included in Figure 1. They visualize the systematic Hale cycle in GCR variation around solar minima. Additionally, they show confirmation for a systematic decreasing trend in both $A_{27}$ and $A_{14}$, which is seen for both NMs. We have calculated the best fit lines to the all five points in each case and included them in the upper panels of Figures 2 and 3. The fits are fairly highly correlated with observations, but the small number of points reduces statistical significance.

The lower panels of Figures 2 and 3 show the detrended values of $A_{27}$ and $A_{14}$, which further clarify the 22-year cycle. We estimated the mean amplitude of the 22-year variation by fitting a 22-year sine function to the five points in each of the lower panels of Figures 2 and 3 . We found very high correlation coefficients (above 0.97) for all other cases except for $A_{27}$ of Apatity, where it was high, but considerably lower than for the other cases. This is due to the rather high value of $A_{27}$ of Apatity during the last solar minimum, which clearly differs from the evolution seen, e.g., in Oulu (see Fig. 2).

The sine amplitudes for $A_{27}$ and $A_{14}$ are $\sim 0.04-0.07$ and 0.06-0.09, respectively. Comparing these to the typical 22-year amplitudes to the mean minima, the relative variations are approximately $15 \%-30 \%$ for $A_{27}$ and $30 \%-45 \%$ for $A_{14}$. Therefore, the 22-year cycle is relatively 

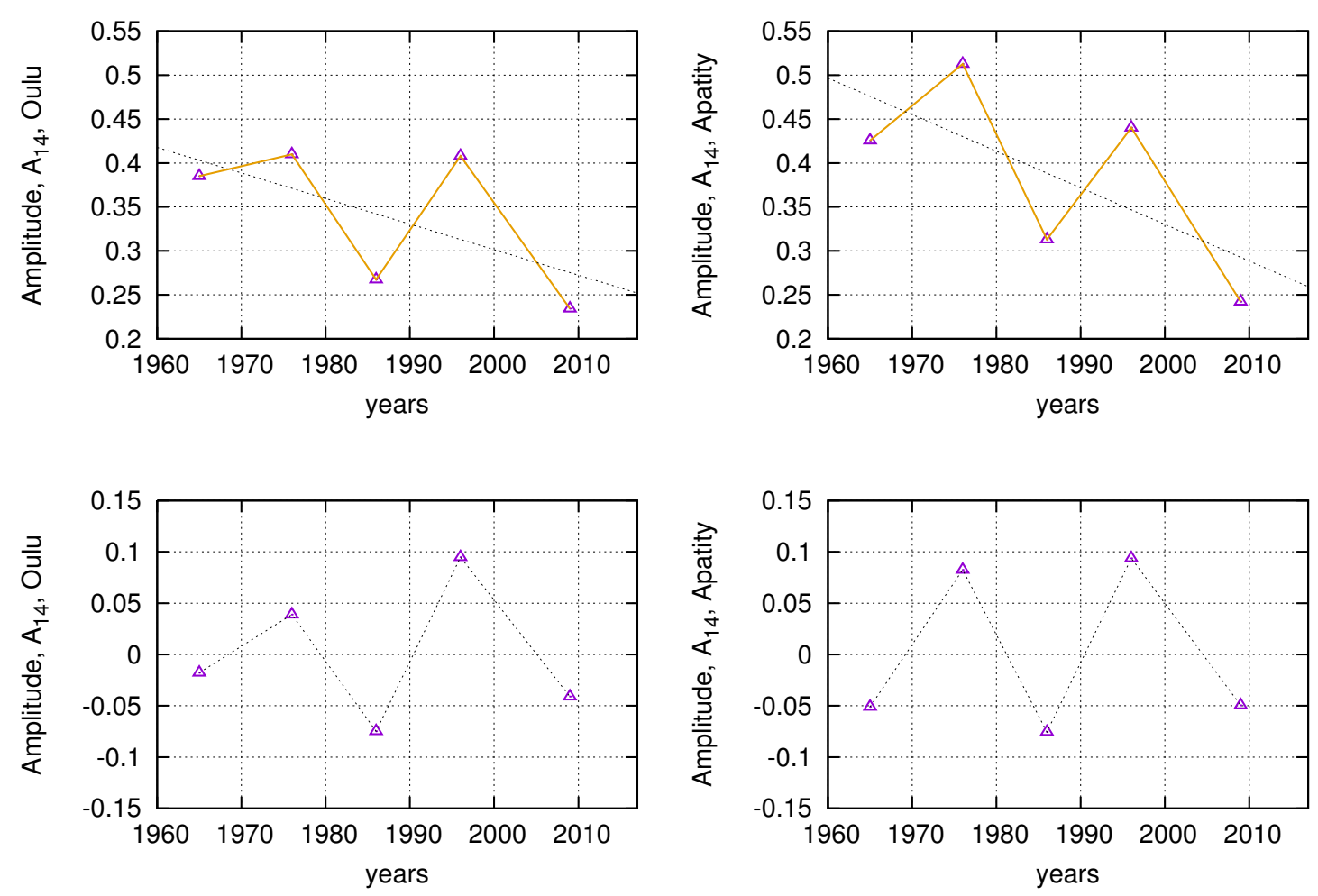

Figure 3: Three-year means amplitude of the second $\left(A_{14}\right)$ harmonic of GCR recurrence for the last five solar minima with linear trend (upper panels) and detrended (lower panels) for Oulu (left panels) and Apatity (right panels) neutron monitor count rates.

considerably stronger in the amplitude of the second harmonic than in the first harmonic.

Figures 1 and 3 show that the undetrended $A_{14}$ experienced their all-time lowest values during the last, prolonged solar minimum. This behavior can be explained by the particular structure of the heliospheric current sheet (HCS) during the last solar minimum. Figure 4 shows the solar wind speed measured by Ulysses during the first [19] and the third [20] fast latitude scan. We can see that the extent of the HCS region (the slow solar wind speed region around the solar equator) in May 2007-November $2007(A<0)$ was considerably larger than in December 1994-May 1995 $(A>0)$ [21]. During the first scan, 1994-1995, Ulysses spacecraft showed clearly separate areas of slow and fast solar wind, but during 2007 the boundary between the fast and slow solar wind regions was less sharp. The extent of the heliospheric current sheet region in 2007 was much broader than in 1994-1995. The large extent of the HCS region in 2007 is connected to the weak polar fields during the declining phase of solar cycle 23 [22]. Thus, the Earth spent more time deep inside the HCS region of slow solar wind during the last solar minimum, leading to rather constant heliospheric conditions and a significant decrease of $A_{27}$ and $A_{14}$. Moreover, $A_{14}$ experienced this damping relatively larger, because it is more probable that the Earth goes outside the HCS region only once during the 27-day rotation period than twice or more often.

Due to the drift pattern during negative polarity minima [23], the GCR particles preferentially 

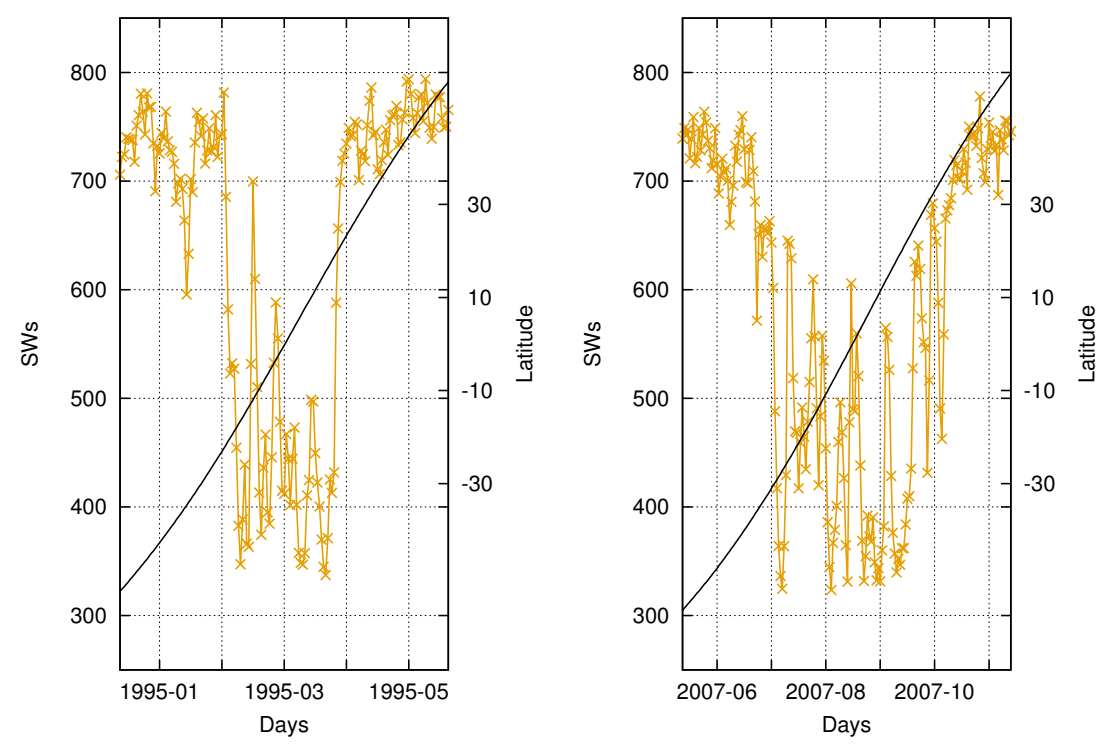

Figure 4: Daily solar wind speed (SWs in km/sec) measured by Ulysses during the first (December 1994May 1995, left panel) and the third (May 2007-November 2007, right panel) fast latitudinal scan. Black dash-dotted curve ( $\mathrm{y}$ - axis on the right) illustrates latitudinal (in degrees) position of the space probe during that period.

drift inward along the HCS at low heliolatitudes, where solar wind speed is low and, therefore, the effect of convection remains small. Rather, the properties of the HCS, in particular its extent, play a leading role at this time. On the other hand, during $A>0$ GCR particles drift over a larger range of heliolatitudes and they also meet faster solar wind, whence convection increases. Thus, GCR particles arriving at the Earth have experienced quite different solar wind conditions during one solar rotation at these times. This increases the rotational variability amplitudes during positive polarity times. These differences in cosmic rays conditions during $A>0$ and $A<0$ polarity times lead to the Hale cycle in the rotation amplitude of GCR recurrence. Accordingly, the related Hale cycle is due to the different drift patterns of GCR and the different causes of modulation during the two polarity times: during $A<0$ the properties of the HCS play the leading role, during $A>0$ the latitudinal variation of solar wind is important.

\section{Conclusions}

We have studied in details the variation of the solar rotation related amplitudes $A_{27}$ and $A_{14}$ of GCR flux. We have quantified the Hale cycle in $A_{27}$ and $A_{14}$ during the previous five solar minima in a robust way and give a physical explanation to the earlier ideas based on the influence of drift to GCR flux at $1 \mathrm{AU}$. We show that the average amplitude of the 22-year cycle is larger, $30-45 \%$ of the mean amplitude for $A_{14}$, than for $A_{27}$ where it is $\sim 15-30 \%$. We conclude that the observed Hale cycle in the solar rotation related variation of galactic cosmic rays is owing to the different drift patterns and different causes of modulation of GCR during the two polarity periods: during the negative the heliospheric current sheet plays the leading role, while during positive the 
heliolatitudinal change of solar wind is more important. We find that the $A_{27}$ and $A_{14}$ amplitudes during the solar cycle minimum epochs depict a declining trend which can be associated with the solar polar magnetic fields weakening during the last four solar cycles [22]. The weakening polar fields lead to a widening of the HCS region, whence the Earth spends more time within the slow solar wind region, decreasing $A_{27}$ and $A_{14}$ during negative polarity times. The weakening of fields culminated during the last solar minimum, decreasing especially the $A_{14}$ amplitude to record low level, thus increasing the related 22-year cycle in $A_{14}$. Ulysses measurements verified that the slow solar wind region was considerably larger during the last solar minimum than during the one earlier. The widening of HCS is relatively more important for the amplitude of the second harmonic $\left(A_{14}\right)$, because it is more likely that Earth visits outside the HCS region only once per solar rotation than twice of more often.

\section{Acknowledgements}

We acknowledge investigators of Apatity, http://pgia.ru/data/nm and Oulu, http://cosmicrays.oulu.fi/ neutron monitors, and the neutron monitor database, http://www.nmdb.eu/. Ulysses data are from http://omniweb.gsfc.nasa.gov. This work was supported by the Center of Excellence ReSoLVE (project No. 272157). A.G. acknowledges The Polish National Science Centre, decision number DEC-2016/22/E/HS5/00406.

\section{References}

[1] S. E. Forbush, On cosmic-ray effects associated with magnetic storms, Terrestrial Magnetism and Atmospheric Electricity (Journal of Geophysical Research) 43 (1938) 203

[2] I. G. Richardson, H. V. Cane, and G. Wibberenz, A 22-year dependence in the size of near-ecliptic corotating cosmic ray depressions during five solar minima, J. Geophys. Res. Space Phys. 104 (1999) 12549-12562

[3] G. E. Hale, F. Ellerman, S. B. Nicholson, and A. H. Joy, The Magnetic Polarity of Sun-Spots, Astrophys. J 49 (1919) 153

[4] M. V. Alania, D. G. Baranov, M. I. Tyasto, and E. S. Vernova, 27-Day variations of galactic cosmic rays and changes of solar and geomagnetic activities, Advances in Space Research 27 (2001) 619-624

[5] A. Gil, and M. V. Alania, 27-day variations of cosmic rays for the minima epochs of solar activity: experimental and 3-D drift modelling results, 27th International Cosmic Ray Conference, 9 (2001) 3725-3728

[6] J. Kota, and J. R. Jokipii, 2001, Recurrent Depressions of Galactic Cosmic Rays in CIRs: 22-Year Cycle, 27th International Cosmic Ray Conference, 9 (2001) 3577-3580

[7] K. Iskra, M. V. Alania, A. Gil, R. Modzelewska, and M. Siluszyk, On Roles of the Stochastic and Regular Heliospheric Magnetic Fields in Different Classes of Galactic Cosmic Ray Variations, Acta Physica Polonica B 35 (2004) 1565

[8] P. Dunzlaff, B. Heber, A. Kopp, et al., Observations of recurrent cosmic ray decreases during solar cycles 22 and 23, Annales Geophysicae 26 (2008) 3127-3138

[9] M. V. Alania, A.Gil, and R. Modzelewska, Study of the 27-day variations of the galactic cosmic ray intensity and anisotropy, Advances in Space Research 41 (2008) 280-286 
[10] W. H. Fonger, Cosmic Radiation Intensity-Time Variations and Their Origin. II. Energy Dependence of 27-Day Variations, Physical Review 91 (1953) 351-361

[11] J. A. Simpson, Cosmic-Radiation Intensity-Time Variations and Their Origin. III. The Origin of 27-Day Variations, Physical Review 94 (1954) 426-440

[12] K. Nagashima, S. Sakakibara, K. Murakami, and I. Morishita, Response and yield functions of neutron monitor, Galactic cosmic ray spectrum and its solar modulation, derived from all the available world-wide surveys, Nuovo Cimento C Geophysics Space Physics C 12 (1989) 173-209

[13] J. A. Simpson, The Cosmic Ray Nucleonic Component: The Invention and Scientific Uses of the Neutron Monitor - (Keynote Lecture), Space Science Rev. 93 (2000) 11-32

[14] D. Xue, and Y. Chen, Solving Applied Mathematical Problems with MATLAB (2008) CRC Press

[15] H. V. Cane, I. G. Richardson, and T. T. von Rosenvinge, Cosmic ray decreases: 1964-1994, J. Geophys. Res. Space Phys. 101 (1996) 21561-21572

[16] I. G. Richardson, and H. V. Cane, Galactic Cosmic Ray Intensity Response to Interplanetary Coronal Mass Ejections/Magnetic Clouds in 1995 - 2009, Solar Physics 270 (2011) 609-627

[17] O. O. Musalem-Ramirez, J. F. Valdes-Galicia, G. Munoz, and E. Huttunen, A catalog of Forbush decreases of the cosmic radiation for the period 1997-2007, Proceedings of the 33rd International Cosmic Rays Conference (2010) 0393

[18] P. Meyer, and J. A. Simpson, Changes in Amplitude of the Cosmic-Ray 27-Day Intensity Variation with Solar Activity, Physical Review 96 (1954) 1085-1088

[19] E. J. Smith, A. Balogh, M. E. Burton, G. Erdös, and R. J. Forsyth, Results of the Ulysses fast latitude scan: Magnetic field observations, Geophys. Res. Lett. 22 (1995) 3325-3328

[20] R. W. Ebert, D. J. McComas, H. A. Elliott, R. J. Forsyth, and J. T. Gosling, Bulk properties of the slow and fast solar wind and interplanetary coronal mass ejections measured by Ulysses: Three polar orbits of observations, J. Geophys. Res. Space Phys. 114 (2009) A01109

[21] I. I. Virtanen, and K. Mursula, Asymmetry of solar polar fields and the southward shift of HCS observed by Ulysses, J. Geophys. Res. Space Phys. 115 (2010) A09110

[22] E. J. Smith, and A. Balogh, Decrease in heliospheric magnetic flux in this solar minimum: Recent Ulysses magnetic field observations, Geophys. Res. Lett. 35 (2008) L22103

[23] J. R. Jokipii, and B. Thomas, Effects of drift on the transport of cosmic rays. IV - Modulation by a wavy interplanetary current sheet, Astrophys. J. 243 (1981) 1115-1122 